\title{
open_iA: A tool for processing and visual analysis of industrial computed tomography datasets
}

\section{Bernhard Fröhler ${ }^{1,2}$, Johannes Weissenböck ${ }^{1,3}$, Marcel Schiwarth ${ }^{1}$, Johann Kastner ${ }^{1}$, and Christoph Heinzl ${ }^{1}$}

1 University of Applied Sciences Upper Austria, Research Group Computed Tomography 2 University of Vienna, Research Group Visualization and Data Analysis 3 TU Wien, Visualization Working Group

DOI: $10.21105 /$ joss. 01185

\section{Software}

- Review ¿

- Repository ¿a

- Archive c

Submitted: 21 December 2018

Published: 21 March 2019

\section{License}

Authors of papers retain copyright and release the work under a Creative Commons Attribution 4.0 International License (CC-BY).

\section{Summary}

open_iA is a platform for visual analysis and processing of volumetric datasets. The main driver behind its development is to provide a common framework for performing visual analytics on industrial Computed Tomography (CT) data. In contrast to general volume visualization or processing software, it offers specialized tools, which address domain-specific analysis scenarios such as porosity determination, fiber characterization and image processing parameter space analysis. The wide range of building blocks, which these tools consist of, facilitate the development of new research prototypes in this application domain. It currently provides a variety of image processing filters, e.g. for noise reduction, segmentation, data type conversion, convolution, geometric transformations, and morphological operations. open_iA is written in C++ using Qt, VKT and ITK, as well as some other open source libraries. open_iA is continuously improved and extended. It is open source and available on GitHub. The core of open iA provides functionality for loading and displaying volumetric datasets in several file formats, as well as support for loading polygonal datasets. A comparison of volumes is facilitated through a magic lens as well as optional position indicators in all open child windows. In addition, it provides a view for showing the image histogram, where also the transfer function used for the slicer views and the $3 \mathrm{D}$ renderer is configured.

open_iA is highly extensible through what we call modules, which makes it an ideal platform for research prototypes and tools. These modules can contain anything from simple image processing filters to complex visual analytics tools. Several publications so far were already based on modules implemented in the open_iA framework. The modules in the following list are included in the open_iA repository:

- DreamCaster (A. Amirkhanov, Heinzl, Reiter, \& Gröller, 2010) is a tool for finding the best scanning parameters in a CT device for a given specimen.

- FiberScout (Weissenböck et al., 2014), later extended to FeatureScout, is a tool for analyzing the properties of collections of similar objects.

- MObjects (Reh, Gusenbauer, Kastner, Gröller, \& Heinzl, 2013) now included in the FeatureScout tool, provides a way to visualize the average shape of a collection of similar objects, such as fibers or pores.

- The 4DCT tools (Amirkhanov et al., 2016) enable exploring multiple CT datasets from different stages of fatigue testing of fiber-reinforced polymers, where one can classify and analyze types of defects and defect formations. 
- Fuzzy Feature Tracking (Reh, Amirkhanov, Heinzl, Kastner, \& Gröller, 2015) provides graphs for tracking the creation, continuation, and merge of defects between different stages of fatigue testing.

- GEMSe (Fröhler, Möller, \& Heinzl, 2016) supports users in finding optimal parameters for their volume segmentation tasks without requiring a ground truth.

- The Porosity Analyzer (Weissenböck, Amirkhanov, Gröller, Kastner, \& Heinzl, 2016) similarly supports users in finding the ideal segmentation algorithm and parameterization when they are determining porosity values, e.g., in fiber-reinforced polymers.

- InSpectr (A. Amirkhanov, Fröhler, Kastner, Gröller, \& Heinzl, 2014) makes it possible to analyze spectral data, e.g., from X-Ray fluorescence spectral tomography, alongside with data from computed tomography for the same specimen.

- Dynamic Volume Lines (Weissenböck, Fröhler, Gröller, Kastner, \& Heinzl, 2019) facilitate the comparison of multiple slightly varying volumetric datasets, by mapping them to $1 \mathrm{D}$ and applying a nonlinear scaling to highlight regions with large differences.

- With MetaTracts (Bhattacharya, Heinzl, Amirkhanov, Kastner, \& Wenger, 2015, Bhattacharya et al. (2017)) one can characterize and analyze fiber bundles as well as weaving patterns in fiber-reinforced polymers.

\section{Acknowledgments}

The work leading to the results shown here was supported by the K-Project for "nondestructive testing and tomography plus" (ZPT+) and by the COMET program of FFG and the federal government of Upper Austria and Styria. It also received funding by the project "Multimodal and in-situ characterization of inhomogeneous materials" (MiCi) by the federal government of Upper Austria and the European Regional Development Fund (EFRE) in the framework of the EU-program IWB2020. Furthermore, it received funding from the FFG BRIDGE early stage project no. 851249, "Advanced multimodal data analysis and visualization of composites based on grating interferometer micro-CT data" (ADAM), as well as from the Research Foundation Flanders (FWO) and the Austrian Science Fund (FWF) project "Quantitative X-ray tomography of advanced polymer composites", under the grant numbers G0F9117N and I3261-N36 respectively. This work was also supported by the project "Interpretation and evaluation of defects in complex CFK structures based on 3D-CT data and structural simulation" (DigiCT-Sim; FFG proj. no. 862015) funded by the federal government of Upper Austria and FFG.

\section{References}

Amirkhanov, A., Amirkhanov, A., Salaberger, D., Gröller, E., Kastner, J., \& Heinzl, C. (2016). Visual analysis of defects in glass fiber reinforced polymers for 4DCT interrupted in situ tests. Computer Graphics Forum, 35(3), 201-210. doi:10.1111/cgf.12896

Amirkhanov, A., Fröhler, B., Kastner, J., Gröller, E., \& Heinzl, C. (2014). InSpectr: Multi-modal exploration, visualization, and analysis of spectral data. Computer Graphics Forum, 33(3), 91-100. doi:10.1111/cgf.12365

Amirkhanov, A., Heinzl, C., Reiter, M., \& Gröller, E. (2010). Visual optimality and stability analysis of 3DCT scan positions. In Proceedings 21st IEEE Visualization Conference (pp. 1477-1486). Salt Lake City, Utah, USA: IEEE. doi:10.1109/TVCG.2010.214

Bhattacharya, A., Heinzl, C., Amirkhanov, A., Kastner, J., \& Wenger, R. (2015). MetaTracts - A method for robust extraction and visualization of carbon fiber bundles in fiber 
reinforced composites. In Procedings 2015 IEEE Pacific Visualization Symposium (pp. 191-198). Hangzhou, China: IEEE. doi:10.1109/PACIFICVIS.2015.7156377

Bhattacharya, A., Weissenböck, J., Wenger, R., Amirkhanov, A., Kastner, J., \& Heinzl, C. (2017). Interactive exploration and visualization using MetaTracts extracted from carbon fiber reinforced composites. IEEE Transactions on Visualization and Computer Graphics, 23(8), 1988-2002. doi:10.1109/TVCG.2016.2582158

Fröhler, B., Möller, T., \& Heinzl, C. (2016). GEMSe: Visualization-guided exploration of multi-channel segmentation algorithms. Computer Graphics Forum, 35(3), 191-200. doi:10.1111/cgf.12895

Reh, A., Amirkhanov, A., Heinzl, C., Kastner, J., \& Gröller, E. (2015). Fuzzy feature tracking: Visual analysis of industrial 4D-XCT data. Computers \& Graphics, 3(1), 177184. doi:10.1016/j.cag.2015.04.001

Reh, A., Gusenbauer, C., Kastner, J., Gröller, E., \& Heinzl, C. (2013). MObjects - A novel method for the visualization and interactive exploration of defects in industrial XCT data. IEEE Transactions on Visualization and Computer Graphics, 19(12), 2906-2915. doi:10.1109/TVCG.2013.177

Weissenböck, J., Amirkhanov, A., Gröller, E., Kastner, J., \& Heinzl, C. (2016). PorosityAnalyzer: Visual analysis and evaluation of segmentation pipelines to determine the porosity in fiber-reinforced polymers. In Proceedings 2016 IEEE Conference on Visual Analytics Science and Technology (pp. 101-110). Baltimore, MD, USA: IEEE. doi:10.1109/VAST.2016.7883516

Weissenböck, J., Amirkhanov, A., Li, W., Reh, A., Amirkhanov, A., Gröller, E., Kastner, J., et al. (2014). FiberScout: An interactive tool for exploring and analyzing fiber reinforced polymers. In Proceedings 2014 IEEE Pacific Visualization Symposium (pp. 153-160). Yokohama, Japan: IEEE. doi:10.1109/PacificVis.2014.52

Weissenböck, J., Fröhler, B., Gröller, E., Kastner, J., \& Heinzl, C. (2019). Dynamic volume lines: Visual comparison of $3 \mathrm{D}$ volumes through space-filling curves. IEEE Transactions on Visualization and Computer Graphics, 25(1), 1040-1049. doi:10.1109/TVCG. 2018.2864510 